\title{
PROJETO DE ESTRUTURA METÁLICA DE PASSARELA PARA TRAVESSIA DE
}

\section{PEDESTRES}

PROJECT OF METALLIC STRUCTURE OF PASSARELA FOR PEDESTRIAN TRANSFER

Edianêz Scheffemacher Ribeiro ${ }^{1}$

Luiz Fernando Gardini²

\section{RESUMO}

Este trabalho apresenta o projeto da estrutura metálica de uma passarela para travessia de pedestres. Expondo os tipos de passarelas existentes e seus elementos estruturais, segundo a ISF - 219 do DNIT. Mostrando também os componentes que formam a estrutura metálica dessa passarela, sendo que dentre eles estão o tabuleiro, a treliça principal, as longarinas, transversinas, entre outros. Os acessos de uma passarela serão apresentados levando em consideração a NBR 9050, que dispõe sobre a acessibilidade em edificações, abrangendo o mobiliário, os espaços e os equipamentos urbanos. Ainda seguindo o que informa esta norma, apresentamos a altura, espessura, distância da parede, corretas para a instalação do corrimão. Visando principalmente a apresentação dos cálculos realizados para que seja projetado corretamente a estrutura metálica, seguindo diversas normas da Associação Brasileira de Normas Técnicas - ABNT. Segundo as normas da ABNT, apresentaremos o cálculo das cargas que agem sobre a estrutura, dentre elas estão: ação do vento, carga móvel, carga permanente, carga variável.

Palavras-Chave: Estrutura. Aço. Passarela. Projeto.

\section{ABSTRACT}

This work presents the design of the metallic structure of a walkway for pedestrians crossing. Exposing the types of existing walkways and their structural

\footnotetext{
${ }^{1}$ Acadêmica do Curso de Engenharia Civil da Universidade Alto Vale do Rio do Peixe (UNIARP).

2 Professor Orientador. Graduado em Engenharia de Produção - Mecânica, pela Universidade do Oeste de Santa Catarina, Pós-Graduado em Engenharia de Produção, pela Universidade do Oeste de Santa Catarina. Pós Graduado em Engenharia de Segurança do Trabalho, pela Universidade do Oeste de Santa Catarina. Docente dos Cursos de Engenharia Civil e Engenharia Mecânica da Universidade Alto Vale do Rio do Peixe (UNIARP).
} 
elements, according to DNF ISF - 219. Also showing the components that form the metallic structure of this catwalk, being among them are the board, the main trellis, the railings, transverse, among others. The accesses of a catwalk will be presented taking into consideration the NBR 9050, which deals with accessibility in buildings, covering the furniture, spaces and urban equipment. Still following what this standard informs, we present the height, thickness, distance of the wall, correct for the installation of the handrail. Aiming mainly the presentation of the calculations made so that the metal structure is correctly designed, following several standards of the Brazilian Association of Technical Standards - ABNT. According to ABNT norms, we will present the calculation of the loads that act on the structure, among them are: wind action, moving load, permanent load, variable load.

Keywords: Structure. Steel. Footbridge. Project.

\section{INTRODUÇÃO}

Este trabalho tem como objetivo apresentar o projeto estrutural em aço de uma passarela para travessia de pedestres. Abordando os principais motivos pelos quais o local de implantação foi escolhido, levando-se em consideração que a localidade apresenta grande fluxo de estudantes e trabalhadores, por se tratar de uma área industrial localizada entre dois bairros, onde estão localizadas várias escolas.

As vantagens de se utilizar o aço como material para a construção da passarela são inúmeras, considerando que o aço apresenta alta resistência estrutural, a vibração e a possíveis choques, possibilita a execução do projeto com mais rapidez, assim como permite vencer grandes vãos.

Para o projeto da estrutura metálica que suportará as cargas da passarela para travessia de pedestres, leva-se em consideração a NBR 8800 (ABNT, 2008), que apresenta as disposições a serem seguidas para a elaboração de Projetos de Estruturas de Aço e de Estruturas Mistas de Aço e Concreto de Edifícios.

O Departamento Nacional de Infraestrutura de Transportes dispõe de um manual, o ISF - 219, que fala apresenta alguns requisitos para a elaboração de um Projeto de Passarela para Pedestres, sendo de relevante importância para a elaboração deste trabalho. 


\section{DESENVOLVIMENTO}

LOCAL

A passarela será instalada nas proximidades da Empresa Trans Rio Sul, localizada na Rodovia Estadual Dr. Lourenço Faoro, SC 350, km 66+700.

\section{NECESSIDADE}

Um dos fatores mais importantes para a definição do local de implantação de uma passarela é o fluxo de pedestres que por ali transitam diariamente. O local escolhido para a implantação fica próximo a estabelecimentos comerciais, indústrias e a bairros essencialmente residenciais, criando assim um fluxo diário muito grande de trabalhadores das indústrias ali implantadas, crianças que frequentam escolas próximas à rodovia, e transeuntes que por ali passam. Existem faixas elevadas para pedestres edificadas ao longo da via, porém, nem sempre os pedestres respeitam e acabam atravessando a rodovia correndo e pulando a barreira de proteção, muitas vezes atrapalhando o fluxo de carros, podendo ocasionar graves acidentes. Para dar melhor mobilidade e acessibilidade aos moradores do entorno é indispensável a implantação de um método de travessia da via, mais adequado e seguro, sendo que a passarela é um dos métodos mais seguros e adequados.

\section{TIPOS DE PASSARELAS}

Segundo o Manual do DNIT ISF - 219, podemos classificar as passarelas em dois tipos diferentes, sendo eles:

- Sobrejacentes: ficam acima das pistas de rolamento, podem ser cobertas ou a céu aberto;

- Subjacentes: ficam abaixo das pistas de rolamento (túneis).

A passarela em questão será do tipo sobrejacente, sendo o mais adequado para a localidade onde será edificada.

Nas passarelas sobrejacentes deverão ser levados em consideração os seguintes dados geométricos e elementos para proteção dos pedestres: 
- Seção horizontal: deve ter um tabuleiro com no mínimo 2 m de largura;

- Seção vertical: guarda-corpo deve ter uma altura mínima de 1 m, podendo ser construído em concreto armado ou aço, deve ser fixado ao vigamento principal do tabuleiro, assegurando uma resistência mínima ao impacto de 80 kgf contra o corrimão e tendo uma cerca com tela de malha de $5 \mathrm{~cm}$, fixada ao guardacorpo, até altura de 2,0 m acima do tabuleiro, na extensão da

- Gabarito vertical: no mínimo de 6,75 m, com referência ao boleto do trilho

- Extensão: deve estender-se em direção transversal e posição superposta ao eixo longitudinal da plataforma do corpo estradal, prolongando-se por $10 \mathrm{~m}$ a partir dos bordos externos da faixa de domínio até as interseções com os respectivos acessos.

Tipos de Elementos Estruturais Construtivos

Ainda Segundo o Manual do DNIT ISF - 219, para as passarelas sobrejacentes, poderemos utilizar os seguintes tipos:

- Estrutura em concreto armado;

- Estrutura metálica, em aço;

Mista combinando os dois elementos.

ESCOLHA DO MATERIAL

Segundo Pinheiro (2005), a utilização do aço como material estrutural apresenta diversas vantagens sobre outros materiais, sendo elas:

- Produção das estruturas com exatidão milimétrica, permitindo um alto controle do produto finalizado.

- Segurança de que as dimensões e propriedades dos materiais estarão corretas;

- Material resistente à vibração e a choques;

- Possibilidade de edificação de obras com mais rapidez e limpeza;

- Permite a desmontagem das estruturas e sua posterior montagem em outro local, caso seja necessário;

- Possui elevada resistência estrutural, permitindo a execução de 
estruturas leves para vencer amplos vãos;

- Possibilita o reaproveitamento dos materiais em estoque, ou mesmo, materiais restantes de outras obras.

\section{RESULTADOS}

PROJETO DA PASSARELA

\section{Considerações Arquitetônicas Gerais}

Dentre os principais elementos constituintes de uma passarela, estão:

\section{Acessos}

Existem basicamente três possíveis tipos de acessos para uma passarela, sendo eles: escadas comuns ou rolantes, rampas ou elevadores (TONIN, 2014). No caso da passarela a ser dimensionada, o acesso para a mesma será feito através de rampas. Facilitando o acesso de todos, e levando em consideração a NBR 9050 Acessibilidade a edificações, mobiliário, espaços e equipamentos urbanos (ABNT, 2004).

a) Rampa de acesso

a.1) Inclinação

Segundo a NBR 9050 (ABNT, 2004), a inclinação da rampa de acesso não deve ultrapassar $8,33 \%$.

A inclinação obtida para a rampa da passarela em questão foi de $8,23 \%$.

Através da multiplicação da altura do desnível x 100, dividido pelo comprimento da rampa. O desnível é de 3,725 m, pois a altura total é de $6,75 \mathrm{~m}$. E o comprimento da rampa será de $41 \mathrm{~m}$ para adequar-se à porcentagem máxima de inclinação.

\section{a.2) Patamares}

A NBR 9050 (ABNT, 2004), prevê um patamar com as dimensões longitudinais obedecendo as da rampa a cada $50 \mathrm{~m}$ de comprimento de rampa. 
Embora o comprimento da rampa da passarela tenha ficado abaixo desse valor, teremos que colocar um patamar pois teremos dois desníveis.

\section{Corrimão}

Obedecendo as definições da NBR 9050 (ABNT, 2004), o corrimão ficará fixado nas barras de suporte. Feito de barras circulares de ferro, com seção circular de $4 \mathrm{~cm}$. Com sua projeção incidindo $10 \mathrm{~cm}$ para cada lado, na largura da rampa. E com as duas alturas recomendadas pela norma, uma a $70 \mathrm{~cm}$ do piso e outra a 90 $\mathrm{cm}$.

\section{Tabuleiros}

No caso desta passarela estaremos utilizando como tabuleiro, uma laje mista entre concreto e chapas de aço galvanizado, mais conhecida como steel deck. Esta laje possui inúmeras vantagens, como: fácil implantação, rapidez de instalação, entre outras. Possui Peso próprio de aproximadamente 0,98 kN (DA SILVA, 2010).

\section{Carregamentos}

\section{Peso do Tabuleiro}

Como já especificado no item 2.5.3 o tabuleiro será edificado atraves de uma laje steel deck, que possui como peso próprio uma carga de 100 kN.

\section{Peso da Treliça}

Através do Sofware Ftool criamos uma treliça tipo, levando em conta as dimenções da parte central da passarela que ficará sobre as vias de rolamento, e precisa ser fechada, para evitar possíveis acidentes. Tendo ela: 15,20 m de comprimento, 2 m de altura, sendo utilizado para ela o perfil de aço W 150,0 x 13,0 que possui $13,0 \mathrm{Kg} / \mathrm{m}$, levando em cosideração suas dimensões teremos um peso aproximado de $8,826 \mathrm{kN}$. 


\section{Carga de Vento}

A partir dos cálculos efetuados, através das fórmulas apresentadas na NBR 6123 (ABNT, 1988), que levam em consideração vários fatores, sobre a obra e sua localização. Chegamos a um valor de carga de vento inexpressível, podendo então ser considerado desprezível.

\section{Carga Móvel}

De acordo com a NBR 7188 (ABNT, 2013) - Carga Móvel Rodoviária e de Pedestres em Pontes, Viadutos, Passarelas e outras Estruturas. A carga móvel é uniformemente distribuída, e aplicada sobre o pavimento situado entre os guardacorpos, sem levar em consideração coeficiente de impacto vertical. Deve-se considerar a carga como sendo: $p=5,0 \mathrm{kN} / \mathrm{m} 2$.

\section{Carga Total}

Nas 3 vigas de 10,10 m de comprimento que ficam sobre a rodovia, localizadas entre os pilares 3 e 4, e nas outras 3 vigas de 10,10 m de comprimento que ficam entre os pilares 4 e 5 , levando em consideração as cargas mencionadas nos itens a cima, é aplicada uma carga distribuída de 23,63 kN, gerando um momento fletor de 50,2 kN.m e uma reação de apoio de 19,88 kN.

$E$ nas 3 vigas que ficam sob os patamares, lembrando que são dois patamares um em cada lado da passarela, totalizando 6 vigas, que possuem um comprimento de 4,60 m, é aplicada uma carga distribuída total de $6 \mathrm{kN}$, a carga será menor pois não se aplica o peso das treliças. Portanto em cada viga será aplicada uma carga distribuída de $2 \mathrm{kN}$, que gera um momento fletor de 5,29 kN.m e as reações de apoio de 4,60 kN.

NBR 9050 (ABNT, 2004) - Acessibilidade a Edificações, Mobiliário, Espaços e Equipamentos Urbanos.

NBR 8800 (ABNT, 2008) - Projeto de Estruturas de Aço e de Estruturas Mistas de Aço e Concreto de Edifícios 


\section{Seleção dos Perfis}

Seguindo as disposições da NBR 8800 (ABNT, 2008), pudemos definir os perfis a serem utilizados na estrutura metálica da passarela para que suportem as cargas aplicadas em sua estrutura, esses perfis estão dispostos na tabela a seguir:

Tabela 1 - Perfis selecionados para suportar as cargas aplicadas nas vigas Carga Suportada

\begin{tabular}{l|l|l|l|l|l}
\hline Elemento & Dimensões & $\begin{array}{l}\text { Carga } \\
\text { Aplicada }\end{array}$ & $\begin{array}{l}\text { Momento } \\
\text { Fletor }\end{array}$ & Perfil & $\begin{array}{l}\text { Carga } \\
\text { Suportada }\end{array}$ \\
\hline Viga 1 & $4,60 \mathrm{~m}$ & $2 \mathrm{kN}$ & $5,29 \mathrm{kN} \cdot \mathrm{m}$ & W $310 \times 21$ & $8,30 \mathrm{kN} \cdot \mathrm{m}$ \\
\hline Viga 2 & $4,60 \mathrm{~m}$ & $2 \mathrm{kN}$ & $5,29 \mathrm{kN} \cdot \mathrm{m}$ & W $310 \times 21$ & $8,30 \mathrm{kN} \cdot \mathrm{m}$ \\
\hline Viga 3 & $4,60 \mathrm{~m}$ & $2 \mathrm{kN}$ & $5,29 \mathrm{kN} \cdot \mathrm{m}$ & W $310 \times 21$ & $8,30 \mathrm{kN} \cdot \mathrm{m}$ \\
\hline Viga 4 & $4,60 \mathrm{~m}$ & $2 \mathrm{kN}$ & $5,29 \mathrm{kN} \cdot \mathrm{m}$ & W $310 \times 21$ & $8,30 \mathrm{kN} \cdot \mathrm{m}$ \\
\hline Viga 5 & $4,60 \mathrm{~m}$ & $2 \mathrm{kN}$ & $5,29 \mathrm{kN} \cdot \mathrm{m}$ & W $310 \times 21$ & $8,30 \mathrm{kN} \cdot \mathrm{m}$ \\
\hline Viga 6 & $4,60 \mathrm{~m}$ & $2 \mathrm{kN}$ & $5,29 \mathrm{kN} \cdot \mathrm{m}$ & W $310 \times 21$ & $8,30 \mathrm{kN} \cdot \mathrm{m}$ \\
\hline Viga 7 & $10,10 \mathrm{~m}$ & $3,93 \mathrm{kN}$ & $50,22 \mathrm{kN} \cdot \mathrm{m}$ & W $360 \times 64$ & $65,92 \mathrm{kN} \cdot \mathrm{m}$ \\
\hline Viga 8 & $10,10 \mathrm{~m}$ & $3,93 \mathrm{kN}$ & $50,22 \mathrm{kN} \cdot \mathrm{m}$ & W $360 \times 64$ & $65,92 \mathrm{kN} \cdot \mathrm{m}$ \\
\hline Viga 9 & $10,10 \mathrm{~m}$ & $3,93 \mathrm{kN}$ & $50,22 \mathrm{kN} \cdot \mathrm{m}$ & W $360 \times 64$ & $65,92 \mathrm{kN} \cdot \mathrm{m}$ \\
\hline Viga 10 & $10,10 \mathrm{~m}$ & $3,93 \mathrm{kN}$ & $50,22 \mathrm{kN} \cdot \mathrm{m}$ & W $360 \times 64$ & $65,92 \mathrm{kN} \cdot \mathrm{m}$ \\
\hline Viga 11 & $10,10 \mathrm{~m}$ & $3,93 \mathrm{kN}$ & $50,22 \mathrm{kN} \cdot \mathrm{m}$ & W $360 \times 64$ & $65,92 \mathrm{kN} \cdot \mathrm{m}$ \\
\hline Viga 12 & $10,10 \mathrm{~m}$ & $3,93 \mathrm{kN}$ & $50,22 \mathrm{kN} \cdot \mathrm{m}$ & W $360 \times 64$ & $65,92 \mathrm{kN} \cdot \mathrm{m}$ \\
\hline
\end{tabular}

Fonte: (O próprio autor)

Tabela 2 - Perfis selecionados para suportar as cargas aplicadas nos pilares

\begin{tabular}{l|l|l|l|l}
\hline Elemento & Dimensões & $\begin{array}{l}\text { Reações de } \\
\text { Apoio }\end{array}$ & Perfil & Carga Suportada \\
\hline Pilar 1 & $3,75 \mathrm{~m}$ & $13,8 \mathrm{kN}$ & W $150 \times 13$ & $71 \mathrm{kN} \cdot \mathrm{m}$ \\
\hline Pilar 2 & $3,75 \mathrm{~m}$ & $13,8 \mathrm{kN}$ & W $150 \times 13$ & $71 \mathrm{kN} \cdot \mathrm{m}$ \\
\hline Pilar 3 & $6,75 \mathrm{~m}$ & $59,64 \mathrm{kN}$ & W200 $\times 26,6$ & $115 \mathrm{KN} \cdot \mathrm{m}$ \\
\hline Pilar 4 & $6,75 \mathrm{~m}$ & $119,28 \mathrm{kN}$ & W200 $\times 31$ & $132,12 \mathrm{kN} \cdot \mathrm{m}$ \\
\hline Pilar 5 & $6,75 \mathrm{~m}$ & $59,64 \mathrm{kN}$ & W200 $\times 26,6$ & $115 \mathrm{kN} \cdot \mathrm{m}$ \\
\hline Pilar 6 & $3,75 \mathrm{~m}$ & $13,8 \mathrm{kN}$ & W $150 \times 13$ & $71 \mathrm{kN} \cdot \mathrm{m}$ \\
\hline Pilar 7 & $3,75 \mathrm{~m}$ & $13,8 \mathrm{kN}$ & W $150 \times 13$ & $71 \mathrm{kN} \cdot \mathrm{m} \cdot \mathrm{m}$ \\
\hline
\end{tabular}

Os perfis a cima selecionados, foram escolhidos através dos cálculos efetuados, e levando em consideração que eles suportam as cargas exigidas da estrutura pela passarela de pedestres.

\section{Fixações e Junta de Ligação do Pilar ao Solo}

Devido as altas cargas atuantes na estrutura, o correto é que as fixações sejam feitas através de solda. Sabendo-se que os as fixações de perfis soldados só 
podem ser projetadas por um profissional de Engenharia Mecânica.

\section{CONCLUSÃO}

Levando-se em consideração tudo que foi apresentado durante o desenvolvimento do presente trabalho, concluímos que a passarela é de extrema importância para os moradores da região onde ela será implantada, pois trará mobilidade, conforto e principalmente segurança para eles.

A estrutura metálica, com qual será dimensionada a passarela, apresenta várias vantagens sobre as outras estruturas, como: reaproveitamento, precisão, rapidez, alta resistência, entre outros.

Dentre as normas que utilizadas para o projeto da estrutura metálica, estão a NBR 8800 (ABNT, 2008), que apresentando as principais diretrizes para o projeto de estruturas de aço e de estruturas mistas de aço e concreto de edifícios, a NBR 6123 (ABNT, 1988), que informa todos os critérios para cálculo da força exercida pelo vento sobre as estruturas, a NBR 7188 (ABNT, 2013), que apresenta as considerações sobre a carga móvel sobre a estrutura, e a NBR 9050 (ABNT, 2004) que dispõe sobre a acessibilidade a edificações, mobiliário, espaços e equipamentos urbanos. Utilizamos a NBR 8800 (ABNT, 2008) como critério norteador pelo fato da inexistência de uma norma específica para projetos como este em estrutura metálica.

A partir dos cálculos efetuados, pudemos chegar nos perfis adequados para essa estrutura. Levando em consideração as NBR's apresentadas nesse trabalho, os perfis metálicos selecionados suportam adequadamente as cargas aplicadas sobre a estrutura da passarela, concluindo assim o projeto da estrutura ideal para ela.

\section{REFERÊNCIAS}

ASSOCIAÇÃO BRASILEIRA DE NORMAS TÉCNICAS. NBR 6123: Forças devidas ao vento em edificações. Rio de Janeiro, 1988.

ASSOCIAÇÃO BRASILEIRA DE NORMAS TÉCNICAS. NBR 7188: Carga Móvel em Ponte Rodoviária e Passarela de Pedestre. Rio de Janeiro, 2013. 
ASSOCIAÇÃO BRASILEIRA DE NORMAS TÉCNICAS. NBR 8800: Projeto de Estruturas de Aço e de Estruturas Mistas de Aço e Concreto de Edifícios. Rio de Janeiro, 2008.

ASSOCIAÇÃO BRASILEIRA DE NORMAS TÉCNICAS. NBR 9050: Acessibilidade a edificações, mobiliário, espaços e equipamentos urbanos. Rio de Janeiro, 2004.

DA SILVA, Raphael. Projeto de Produção para Construção Metálica Aplicado em Lajes Mistas Steel Deck. São Paulo, 2010.

DEPARTAMENTO NACIONAL DE INFRAESTRUTURA DE TRANSPORTES. ISF219: Projeto de Passarela para Pedestres. DNIT, 2015.

PINHEIRO, Antônio Carlos da Fonseca Bragança. Estruturas metálicas: cálculos, detalhes, exercícios e projetos. Edgard Blücher, 2005.

PONTES FILHO, Glauco. Estradas de rodagem: Projeto geométrico. IPC BRASIL,1998. 